\title{
Multistage Fault Feature Extraction of Consistent Optimization for Rolling Bearings Based on Correlated Kurtosis
}

\author{
Long Zhang $\mathbb{D}^{D}$, Binghuan Cai, Guoliang Xiong, Jianmin Zhou, Wenbin Tu, and Yinquan Yu
}

School of Mechatronics \& Vehicle Engineering, East China Jiaotong University, Nanchang 330013, China

Correspondence should be addressed to Long Zhang; longzh@126.com

Received 7 August 2020; Revised 29 August 2020; Accepted 16 September 2020; Published 12 October 2020

Academic Editor: Changqing Shen

Copyright ( $\odot 2020$ Long Zhang et al. This is an open access article distributed under the Creative Commons Attribution License, which permits unrestricted use, distribution, and reproduction in any medium, provided the original work is properly cited.

\begin{abstract}
Fault diagnosis of rolling bearings is not a trivial task because fault-induced periodic transient impulses are always submerged in environmental noise as well as large accidental impulses and attenuated by transmission path. In most hybrid diagnostic methods available for rolling bearings, the problems lie in twofolds. First, most optimization indices used in the individual signal processing stage do not take the periodical characteristic of fault transient impulses into consideration. Second, the individual stages make use of different optimization indices resulting in inconsistent optimization directions and possibly an unsatisfied diagnosis. To solve these problems, a multistage fault feature extraction method of consistent optimization for rolling bearings based on correlated kurtosis $(\mathrm{CK})$ is proposed where maximum correlated kurtosis deconvolution (MCKD) is employed to attenuate the influence of transmission path followed by tunable $Q$ factor wavelet transform (TQWT) to further enhance fault features by decomposing the preprocessed signals into multiple subbands under different $Q$ values. The major contribution of the proposed approach is to consistently use CK as an optimization index of both MCKD and TQWT. The subband signal with the maximum CK which is an index being able to measure the periodical transient impulses in vibration signal yields an envelope spectrum, from which fault diagnosis is implemented. Simulated and experimental signals verified the effectiveness and advantages of the proposed method.
\end{abstract}

\section{Introduction}

Rolling element bearings as a significant component was widely employed in modern mechanical manufacturing systems [1-3]. However, unexpected localized defect may inevitably occur on them due to harsh working conditions [4]. If they are not detected in the early stage, the local defects could lead to significant economic losses and even catastrophes [5]. Therefore, fault detection and diagnosis of bearings play an important role in maintaining the availability and reliability of the mechanical system during the past decades [6,7].

Vibration signal analysis is a commonly used approach since it contained rich information of bearing health conditions $[8,9]$. When localized defects occur on rolling bearings, they will induce periodic transient impacts repeating at the period of the fault, which could trigger the resonant frequencies of the entire mechanical system $[10,11]$. However, at the early stage, weak periodic transient impulses indicating the fault characteristic are usually overwhelmed by harsh background noise, large accidental impulses, and transmission path [12]. Hence, effectively extracting the periodic impact feature by using advanced signal processing techniques is a key task for fault diagnosis of rolling element bearings [13-15].

The raw signals collected by sensors represent the convolution between the periodic transient impulses caused by bearing faults and the transmission function of the system composed of bearing and bearing housing [16]. Minimum entropy deconvolution (MED) was first proposed by Wiggins et al. in 1970 [17] and has successful applications in mechanical fault detection and diagnosis. To eliminate the influence of the system transmission path, MED is used to deconvolute periodic transient impulse signatures from a machine vibration signal by looking for an optimal filter coefficient, and then fault diagnosis is realized by envelope spectrum analysis [18-21]. However, the kurtosis serving as the optimizing index of MED cannot consider the 
characteristic of periodic fault impacts because it is very sensitive to large accidental impulses [22]. In consequence, it tends to yield a single impulse, rather than periodic transient impulses repeating at the period of the fault [23]. Therefore, the result of MED may be unsatisfactory to detect the fault of rolling bearing in the presence of accidental impulses with high amplitude [24]. Subsequently, a new method called the maximum correlated kurtosis deconvolution (MCKD) which takes the periodicity of the fault into account was designed by McDonald [25]. To successfully detect fault correlated impulses of rolling bearing and minimize the influence of the transmission path, Lyu et al. [26] introduced a new method for the extraction of early fault impulse in gearboxes by the hybrid usage of MCKD and quantum genetic algorithm. Although a lot of successful applications have been reported, the predetermination of key parameters of MCKD, like the fault period $T$, depends heavily on prior knowledge. These parameters largely affect the final deconvolution results and thus decide the performance of MCKD in the fault detection and diagnosis.

The periodic transient impulses due to early faults in rolling bearings are often weak especially at the early fault stage [27]. Moreover, such impulses are often submerged by heavy background noise and large accidental impact apart from the transmission path. To eliminate the majority of the noise and interference, the resonance demodulation technique employs a band-pass filter to filter out fault-induced vibrations around one resonance frequency of bearing systems [28]. Then the fault diagnosis is performed by the envelope spectrum [29]. Antoni et al. [30] proposed the fast kurtogram based on 1/3 binary filter banks, in which the frequency band with the maximum kurtosis in the time domain of the filtered signal serves the optimal band-pass filter. However, the kurtogram suffers the same shortcomings as MED since the kurtosis value employed to evaluate the results is too sensitive to handle large accidental impulse. To solve this issue, Zhang et al. [31] introduced an improved kurtogram method combining correlated kurtosis and wavelet packet decomposition to detect the bearing fault. Turntable $Q$ factor wavelet transform (TQWT) which is an improved wavelet transform was originally proposed by Selesnick [32]. A series of wavelet basis functions can be obtained to filter out interference component by adjusting the $Q$ factor, and then the performance of wavelet transform will be improved in the field of denoising, classification, and signal separation. From then on, TQWT theory has been playing an increasingly important role in the rolling bearing fault diagnosis. However, since the selection of a proper $Q$ factor is a challenging task and has a significant influence to obtain the optimal frequency band, an appropriate frequency band evaluation index is a key issue to guarantee the ultimate result of denoising.

In recent years, there is a trend to jointly use multiple signal processing techniques for vibration signal denoising to enhance fault impulses. Shang et al. [33] explored a new approach to detect rolling bearing fault, where multipoint kurtosis optimal minimum entropy deconvolution adjusted (MOMEDA) is adopted to eliminate the influence of transmission path while improved empirical wavelet transform (IEWT) is used to reduce the interference of noises, and then the optimal component with the maximum kurtosis is obedient to envelope spectrum. Zhang et al. [34] decomposed original signals using improved compound interpolation envelope (ICIE) LMD, and the kurtosis value was employed as the optimization index to select the best component which is further processed by the kurtogram method. Li et al. [35] adopted intrinsic feature-scale decomposition (ICD) to decompose raw signals into a serial of subfrequency bands, TQWT is applied to the component with maximum kurtosis, and then the characteristics frequency ratio (CFR) was used to select the optimal TQWT component. Ma et al. [36] introduced a method combining frequency band extraction and ITQWT for detection of fault transient impulses of rolling bearing. About this method, kurtosis was used to select the optimal $Q$ factor of TQWT prior to the frequency analysis. In the above literatures, multiple signal processing methods are employed together to extract fault feature of bearings. Although the performance has been improved compared with using only one single signal processing method in the case of bearing fault diagnosis, there are still some problems in detecting periodic impulses from heavy background noise for bearing fault identification. One of these problems is that most optimization indices like kurtosis used in individual signal processing stage do not take the periodical characteristic of fault transient impulses into consideration. Another problem is that the individual stages make use of different optimization indices, resulting in inconsistent optimization directions and thus an unsatisfied diagnosis.

To overcome the above two drawbacks and further explore the advanced signal processing method for detecting periodic transient impulses from the heavy interference to implement fault diagnosis of rolling bearing, a multistage fault feature extraction method is proposed based on MCKD and TQWT in this paper, of which the major contribution is the consistent use of correlated kurtosis (CK) as an optimization index of both MCKD and TQWT. Being different from kurtosis, CK can reflect the periodic transient impulse feature. In the proposed method, MCKD is applied to the original vibration signal to initially reduce the effect of signal transmission path and highlight weak impacts to a certain extent. The signal preprocessed by MCKD is subsequently subjected to TQWT to further enhance fault-related characteristics by decomposing the preprocessed signals into multiple subbands under different $Q$ values. The optimal component with the maximum $\mathrm{CK}$ will yield an envelope spectrum, from which fault diagnosis is implemented.

The remainder of this paper is organized as follows: Section 2 describes the basic principle and the disadvantage of MCKD. Section 3 presents a brief description of TQWT. The detailed procedure of the proposed method is illustrated and the simulation experiment is performed in Section 4. The proposed method is further tested to detect with the strong interference signal and the early stage fault of rolling bearing in Section 5. Finally, the conclusions are drawn in Section 6. 


\section{Maximum Correlated Kurtosis Deconvolution}

MED which is a commonly used deconvolution technology was firstly put forwarded by Wiggins. In the past decade, it has been extensively exploited to sharpen the fault impulse signals in rotating parts such as rolling bearings [17]. MED takes maximum kurtosis instead of minimum entropy as the optimization index to obtain an optimal filter to deconvolute the original fault signals. However, MED is susceptible to large accidental impulses because of the kurtosis used as the optimization index. To ensure that periodic transient impulses caused by faults can be extracted effectively, a new index called correlation kurtosis was proposed, which is suited to measure periodic impulses. CK of $M$ shift can be shown as

$$
\mathrm{CK}_{M}\left(T_{S}\right)=\frac{\sum_{n=1}^{N}\left(\prod_{m=0}^{M} y_{n-m T_{S}}\right)^{2}}{\left(\sum_{n=1}^{N} y_{n}^{2}\right)^{M+1}}
$$

For $T_{S}=0, M=1$, the CK becomes the kurtosis in MED. $M$ expresses the order of shift, and $T_{S}$ is the sampling points to iteration period, namely:

$$
T_{\mathrm{s}}=f_{\mathrm{s}} \cdot T
$$

where $f_{\mathrm{s}}$ and $T$ represent the sampling frequency and failure period, respectively.

The MCKD method is a new generation of deconvolution technology derived from MED by McDonald [25]. The essence of MCKD is that CK instead of kurtosis acts as the measure index, and thus the periodic characteristics of bearing local faults can be fully considered. Through the iterative operation, an adverse filter can be obtained [23]. This method has proven able to relieve the effect of transmission path effectively and thus enhances the periodic impacts. When rolling elements run on local faults, periodical impulses are produced. However, the signals collected by using sensors are submerged by the heavy background noise and attenuated by the transmission path, which can be represented by

$$
x=h \cdot y+e_{n}
$$

where $h$ represents the transmission path response, $y$ is the fault signature, and $e_{n}$ denotes the environmental noise.

By searching for a filter maximizing the $\mathrm{CK}$ of the deconvoluted signal, the MCKD algorithm is introduced to recover the periodic transient impacts $y$ in the sensorcollected signal $x$ by minimizing the transmission path effect, to achieve noise reduction and highlight the periodic fault characteristics. This process is called deconvolution, and a formula of inverse filtering is shown as

$$
y=f \cdot x=\sum_{k=1}^{L} f_{k} x_{n-k+1},
$$

where $x$ and $y$ are input and output signals, respectively, $N$ denotes the number of samples of the input signal, $f$ represents the filter coefficients, and $L$ is the length of the filter.

The optimization expression is given by

$$
\max _{f} \mathrm{CK}_{M}\left(T_{S}\right)=\max _{f} \frac{\sum_{n=1}^{N}\left(\prod_{m=0}^{M} y_{n-m T_{S}}\right)^{2}}{\left(\sum_{n=1}^{N} y_{n}^{2}\right)^{M+1}},
$$

where $f$ is the filter and $M$ represents the number of shifts.

The iteration expression of the filter coefficients is presented by

$$
\xi_{f}=\frac{|y|^{2}}{(M+1)|\beta|^{2}}\left(X_{0} X_{0}^{T}\right)^{-1} \sum_{m=0}^{M} X_{m T} \alpha_{m},
$$

where $X_{0} X_{0}^{T}$ indicates the Toeplitz autocorrelation matrix of $x$ and $\left(X_{0} X_{0}^{T}\right)^{-1}$ is supposed to exist. The superscript $T$ represents the transpose operation:

$$
\begin{aligned}
X_{r} & =\left[\begin{array}{cccc}
x_{1-r} & x_{2-r} & \cdots & x_{N-r} \\
0 & x_{1-r} & \cdots & x_{N-1-r} \\
\vdots & \vdots & \ddots & \vdots \\
0 & 0 & \cdots & x_{N-L-r+1}
\end{array}\right]_{L \times N}, \\
\alpha_{m} & =\left[\begin{array}{ccc}
y_{1-m T}^{-1}\left(y_{1}^{2}, y_{1-T}^{2}, \ldots, y_{1-m T}^{2}\right) \\
y_{N-m T}^{-1}\left(y_{N}^{2}, y_{N-T}^{2}, \ldots, y_{N-m T}^{2}\right)
\end{array}\right]_{N \times 1}, \\
\beta & =\left[\begin{array}{ccc}
y_{1} y_{1-T_{S}} & \cdots & y_{1-M T_{S}} \\
\vdots & \vdots & \vdots \\
y_{N} y_{N-T_{S}} & \cdots & y_{N-M T_{S}}
\end{array}\right]_{N \times 1}, \\
r & =\left[\begin{array}{llll}
0 & 2 T & \cdots & m T
\end{array}\right] .
\end{aligned}
$$

Through the above description, the procedure of the MCKD algorithm is presented as follows:

Step 1: load the original data collected by using the acceleration sensor, and determine the sampling points of iteration period

Step 2: calculate $X_{0} X_{0}^{T}$ and $\left(X_{0} X_{0}^{T}\right)^{-1}$ according to the input signal

Step 3: set the initial filter coefficient $\xi_{f}=\left[\begin{array}{llllllll}0 & 0 & \cdots & 1 & -1 & \cdots & 0 & 0\end{array}\right]^{T}$

Step 4: calculate the deconvoluted signal $y$ according to (4)

Step 5: calculate $X_{r}, \alpha_{m}$, and $\beta$ according to $y$ Step 6: compute the new filter coefficient $\xi_{f}$ from (6) Step 7: calculate the iteration error according to the following formula: 


$$
e=\frac{\left(f^{(1)-\mu f^{(0)}}\right)}{\mu f^{(0)}} .
$$

The calculation is terminated as the iteration error is smaller than the threshold value. Otherwise, repeat the process from Step $3[37,38]$.

\section{Theory of Tunable Q-Factor Wavelet Transform}

3.1. Tunable Q-Factor Wavelet Transform. As a new wavelet transform, TQWT was proposed by Selesnick in 2011 [32]. Compared with the traditional constant $Q$ factor wavelet transform, the remarkable feature of TQWT is that it can construct different basis functions by adjusting the $Q$ value to achieve the best matching with the fault features to be extracted. The $Q$ factor was expressed by the ratio of central frequency to bandwidth, as shown in equation (8):

$$
Q=\frac{f_{c}}{B_{W}},
$$

where $f_{\mathrm{c}}$ is the central frequency and $B_{W}$ is the bandwidth [39].

The key of traditional wavelet transform is to select an appropriate wavelet basis function. However, the selection of $Q$ factor of TQWT determines the matching degree between wavelet basis functions and fault features. Figure 1 depicts the decomposition and reconstruction processes of a threelayered TQWT.

In Figure $1, H_{0}(\omega)$ and $H_{1}(\omega)$ denote the frequency response functions of low-pass and high-pass filters, respectively. $H_{0}^{*}(\omega)$ and $H_{1}^{*}(\omega)$ represent the complex conjugates of $H_{0}(\omega)$ and $H_{1}(\omega)$, respectively. $c^{j}$ and $w^{j}$ denote the scale coefficients and wavelet coefficients of wavelet transform, respectively. In consideration of perfect reconstruction, $H_{0}(\omega)$ and $H_{1}(\omega)$ are as follows:

$$
\begin{aligned}
& H_{0}(\omega)= \begin{cases}1, & |\omega| \leq(1-\beta) \pi, \\
\theta\left[\frac{\omega+(\beta-1) \pi}{\alpha+\beta-1}\right], & (1-\beta) \pi<|\omega|<\alpha \pi,\end{cases} \\
& H_{1}(\omega)=\left\{\begin{array}{ll}
0, & \alpha \pi \leq|\omega| \leq \pi, \\
\theta\left[\frac{\alpha \pi-\omega}{\alpha+\beta-1}\right], & (1-\beta) \pi<|\omega|<\alpha \pi, \\
1, &
\end{array}\right]
\end{aligned}
$$

where $\omega$ is the angular frequency and $\theta$ is defined as

$$
\theta(v)=0.5(1+\cos v) \sqrt{2-\cos v}, \quad|v| \leq \pi .
$$

3.2. The Problem of Parameters Selection with TQWT. $Q$ factor and redundancy $r$ have a direct relationship with highpass scaling parameter $\alpha$ and low-pass scaling parameter $\beta$, as in equation (11) [40]:

$$
\begin{aligned}
& \alpha=1-\frac{\beta}{r}, \\
& \beta=\frac{2}{Q+1} .
\end{aligned}
$$

Equations (12) and (13) give the central frequency $f_{c}$ and bandwidth $B_{W}$ of the filter bank in the $j$-th layer, respectively [41]:

$$
\begin{aligned}
& f_{\mathrm{c}}=\alpha^{j} \frac{2-\beta}{4 \alpha} F_{\mathrm{s}}, \\
& B_{w}=\frac{1}{4} \beta \alpha^{j-1} F_{\mathrm{s}},
\end{aligned}
$$

where $j$ denotes the number of decomposition layers and $F_{\mathrm{s}}$ is the sampling frequency. In addition, the largest decomposition layer $J_{\max }$ was obtained as follows [32]:

$$
J_{\max }=\frac{\log (N / 4(Q+1))}{\log ((Q+1) / 4(Q+1-(2 / r)))},
$$

where $\lfloor\cdot\rfloor$ denotes a round-down integer. $N$ represents the data length. For instance, when $Q=1, r=3$, and $N=10,000$, the maximum decomposition level $J_{\max }=17$, while in the case of $Q=3, J_{\max }=35$.

The decomposition performance of TQWT is determined by $Q$ factors, the redundancy $r$, and the number of maximum decomposition levels $J_{\max }$. Moreover, $r \geq 3$ is recommended in Ref. [39]. In order to ensure an excellent computational efficiency for TQWT, $r$ is set to 3 in the present research. Figure 2(a) implies that the time-domain signals of TQWT wavelet are symmetrical and approximately translation invariant. The number of filters is ensured by parameter $J_{\max }$. Meanwhile, different $(Q, r)$ will yield different wavelet basis functions. For $Q=3$, it is noted that the duration of wavelet basis function gets longer as the decomposition layers raise in Figure 2(a). The frequency responses of the first 10 decomposition layers are displayed in Figure 2(b), which represents a group of nonconstant band-pass filters with the central frequency gradually decreasing since the frequency range of the filters of the first 10 layers cover $\left[\begin{array}{ll}0.05 & 0.5\end{array}\right] \times$ sampling frequency. The subband signals resulted from such layers will be considered, which is conducive to decrease the computation intense. However, $Q$ factors affecting the performance of TQWT rely heavily on the prior knowledge of operators. Therefore, an indicator is required to determine the optimal $Q$ factors to enhance the fault transient impulse signatures.

\section{The Proposed Fault Diagnosis Method}

4.1. The Fault Feature Extraction Based on MCKD and TQWT. A multistage fault feature extraction method of consistent optimization for rolling bearings based on correlated kurtosis $(\mathrm{CK})$ is presented to detect the periodic impact features 


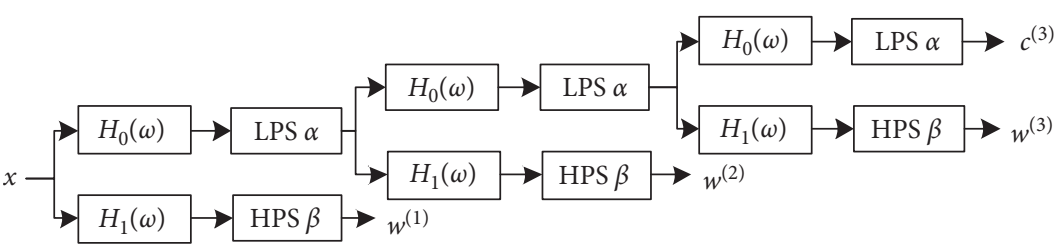

(a)

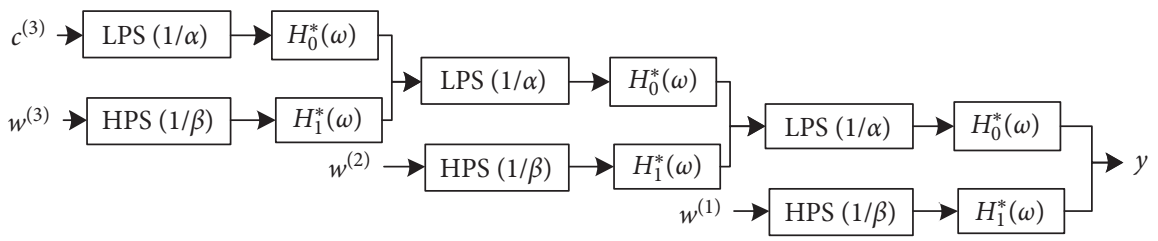

(b)

FIGURE 1: Three-layer filter banks: (a) decomposition filter banks; (b) reconstruction filter banks.

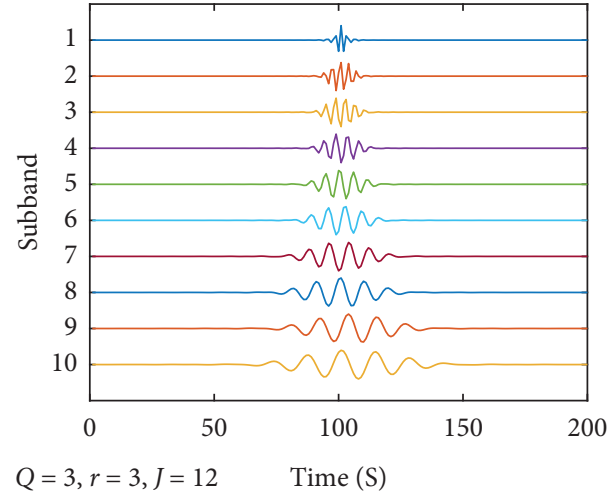

(a)

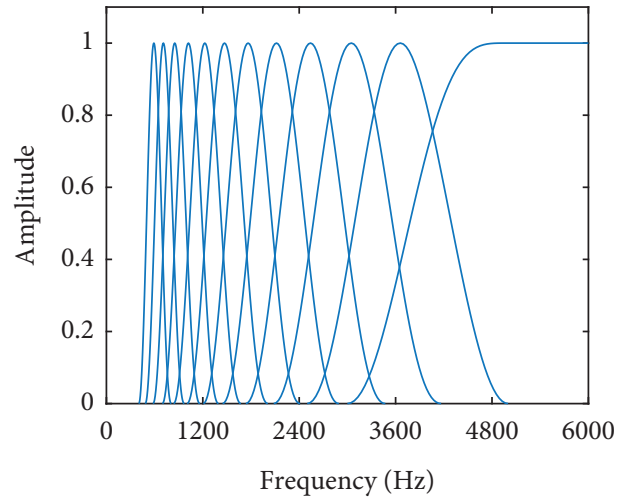

(b)

Figure 2: (a) The time-domain signals and (b) the frequency responses of wavelets in TQWT.

of incipient faults of rolling bearings from harsh background noises and large accidental impulses and mitigate the effect of transmission path. Figure 3 illustrates the procedure of the proposed approach.

(1) The candidate range of $T$ is determined according to the corresponding periods of the inner-race, outerrace, and the rolling element faults

(2) Apply MCKD to deconvolute the original signal for each $T$ obtained in Step (1), resulting in a set of preprocessed signals

(3) Compute the CK value of each deconvoluted signal, and select the optimal $T$ pertinent to maximum $\mathrm{CK}$ to deconvolute the raw signal

(4) Determine the range of $Q$ factor and perform the TQWT on the signal processed by the MCKD with optimal $T$ to obtain a set of wavelet coefficients and scale coefficients corresponding to various $Q$ factors

(5) Reconstruct the first 10 layers of subband signals for all $Q$ factors

(6) Calculate the CK value of each reconstructed subband signals for each $Q$ factor and pave a $2 \mathrm{D}$ map of TQWT
(7) Pick out the optimal component having the largest $\mathrm{CK}$ value in the $2 \mathrm{D}$ map

(8) The optimal component is then subjected to envelope analysis, and fault diagnosis is achieved

4.2. Algorithm Validation with Simulated Signal. The localized defect of rolling bearings is simulated by a vibration model which can be used to verify the approach present in this paper:

$$
\left\{\begin{array}{l}
x(t)=\sum_{i=1}^{M} A_{i} s_{i}\left(t-i T-\tau_{i}\right)+\sum_{j=1}^{M 1} D_{j} s_{j}(t-j T)+n(t), \\
A_{i}=A_{0} \cos \left(2 \pi Q t+\varphi_{A}\right)+C_{A}, \\
s(t)=e^{-B_{i} t} \sin \left(2 \pi f_{n} t+\varphi_{w}\right),
\end{array}\right.
$$

where the first part of $x(t)$ indicates the periodic fault components excited by the localized defect; $A_{i}$ and $A_{0}$ represent the amplitude modulation component and its amplitude, respectively; $C_{A}$ is a constant bias; $s(t)$ shows the impulse response function of the mechanical system; $T$ is the periodic of fault impulses; $B_{i}$ represents the damping 


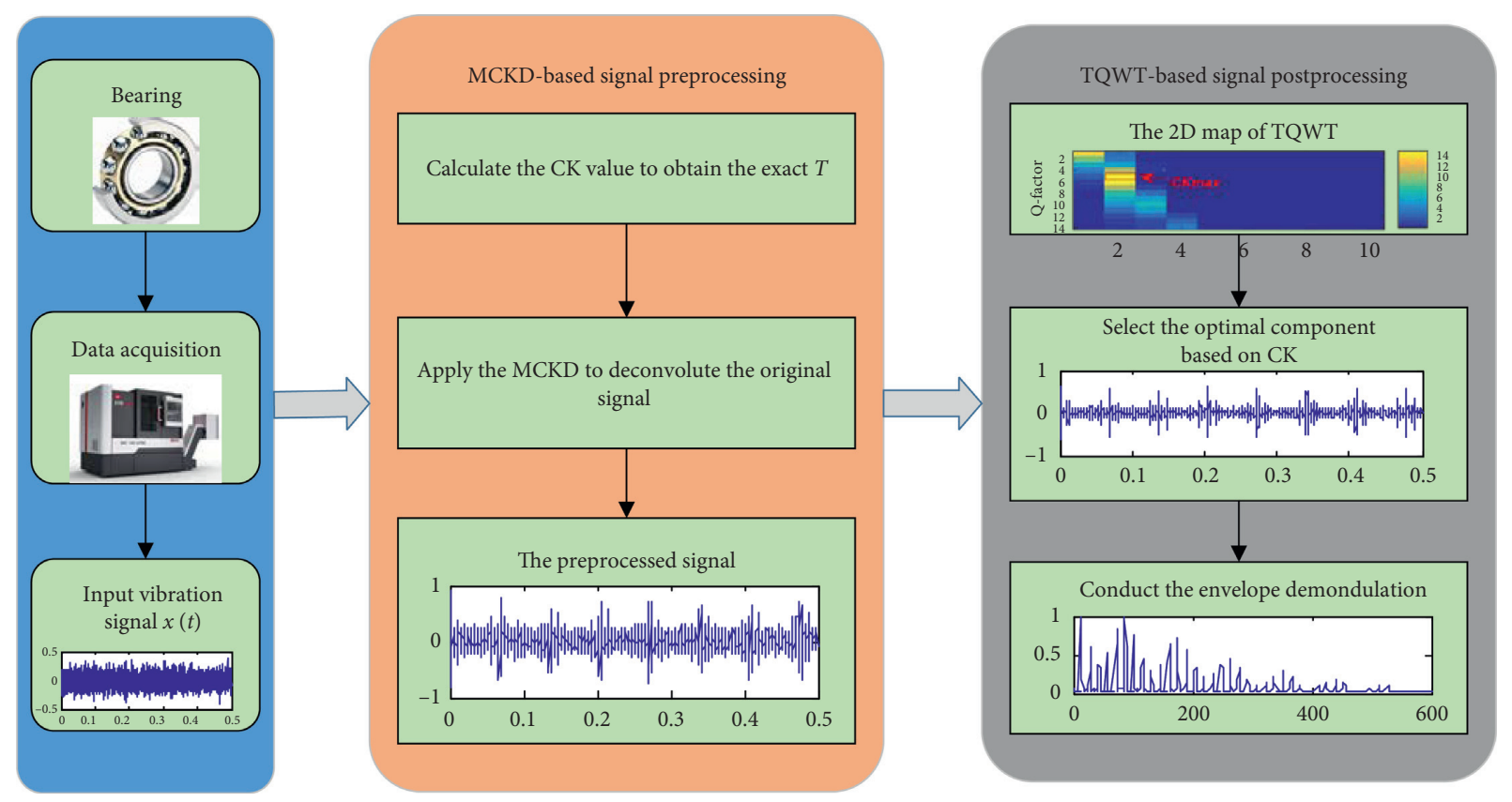

FIgURe 3: Procedure of the proposed approach.

coefficient; the natural frequency can be represented by $f_{n}$; and the white Gaussian noise can be expressed by $n(t)$.

It is the fact that the vibration signals acquired by the sensor may contain large accidental impulses in the actual operation of bearings aside from periodic fault transient impulses and background noise. To better demonstrate that the periodicity of the transient impulses can be extracted by our method sufficiently, considering the external knocks will produce large accidental impact, its simulated signal will be added in the second part of $x(t)$.

An inner-race localized fault is assumed in the simulated signal, whose sampling rate (i.e., $f_{\mathrm{s}}$ ) is $20480 \mathrm{~Hz}$. Its fault characteristic frequency (FCF) is set $90 \mathrm{~Hz}$, and natural frequency produced by impulse is set $3500 \mathrm{~Hz}$. The data length $L$ is set 8000 . Figure 4(a) displays the simulated signal. In addition, to make the simulation signal closer to the vibration signal induced by the actual operation condition of the bearing, the Gaussian random noise with an amplitude of $0.4 \mathrm{~mm} / \mathrm{s}^{2}$ is added to the simulated fault impact signal, and the compound signal is represented in Figure 4(b). Moreover, to verify that kurtosis is susceptible to high amplitude accidental shocks, a sinusoidal signal with an amplitude of 10 and a frequency at $1500 \mathrm{~Hz}$ is artificially added in the range of No. 1000-1060 points as shown in Figure 4(c). It can be noted that the fault transient impulses are submerged by Gaussian background noise and highamplitude sinusoidal impact. Then, from the envelope spectrum of the simulated signal shown in Figure 4(d), there is no obvious fault characteristic frequency component. Consequently, finding an effective fault impulse extraction approach is of significance.

For comparison, the kurtogram approach is employed to process the simulated signal in Figure 3(c). The decomposition layer of the spectral kurtosis is set to 3 due to the requirement on the bandwidth of the filter and the maximum fault characteristic frequency (bandwidth $>3 \times$ maximum FCF). The $2 \mathrm{D}$ map of kurtogram is displayed in Figure 5(a). The optimum parameters of the filter frequency band are that central frequency is $1900 \mathrm{~Hz}$ and bandwidth is $800 \mathrm{~Hz}$, which covers exactly the frequency of $1500 \mathrm{~Hz}$ of the artificially added sinusoidal interference impulse. The envelope and envelope spectrum of the filtered signal resulted from Kurtogram are displayed in Figures 5(b) and 5(c), respectively. The envelope spectrum fails to detect any useful fault information because kurtosis does not take the periodic characteristic of fault impacts into consideration. The results imply that occasional impacts of high amplitude could lead to a wrong selection of filter frequency band.

The approach proposed is used to process the simulated signal. First, the range of the parameter $T$ can be set [220, 280] according to the theoretical values of fault period $T$ corresponding to the inner-race, outer-race, and rolling element fault. MCKD is then employed to deconvolute the simulated signal with the optimal period $T=226$ which is determined by the criterion of maximum CK and approximates to the actual inner-race fault period $T=227$. The fault-related transient impulses of the rolling bearing are initially enhanced as shown in Figure 6(a), but it is not equivalent to detect the inner-race defect. Second, the range of $Q$ factor is set to $[1.0,3.0]$ with an increase of 0.1 . Then, the MCKD preprocessed signal will be decomposed into a series of wavelet coefficients employing TQWT, and the first 10 layers of wavelet coefficients are reconstructed. The CK map of TQWT in Figure 6(d) displays the CK values of each subband component. The optimal reconstructed component is obtained based on the principle of maximizing CK value. The corresponding optimal filter exactly covers the natural frequency $3500 \mathrm{~Hz}$, and the frequency of the sinusoidal interference with $1500 \mathrm{~Hz}$ is avoided. This result shows that 


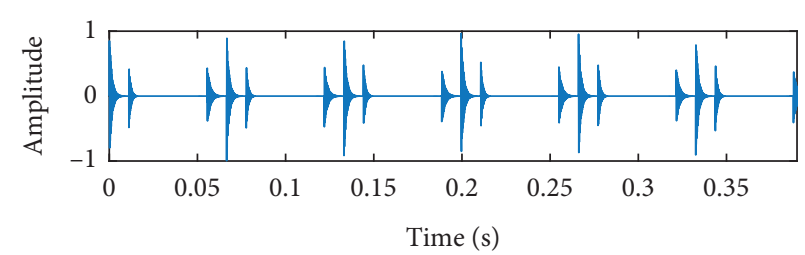

(a)

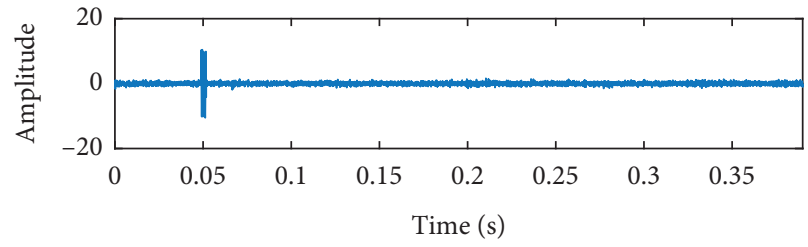

(c)

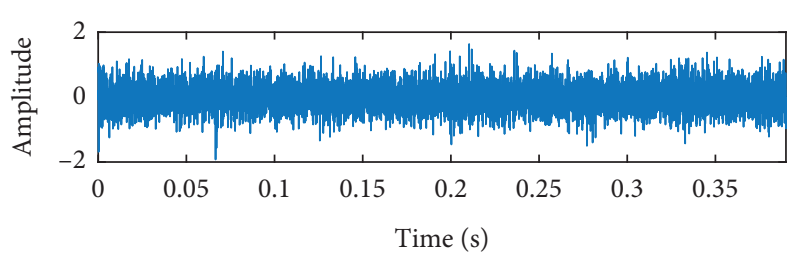

(b)

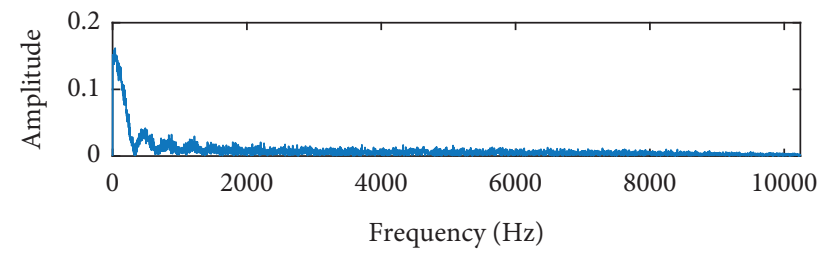

(d)

Figure 4: (a) Periodic transient impulses of inner-race defect; (b) signal with added Gaussian noise; (c) signal with added noise and large accidental impulses; (d) Hilbert envelope spectrum of (c).

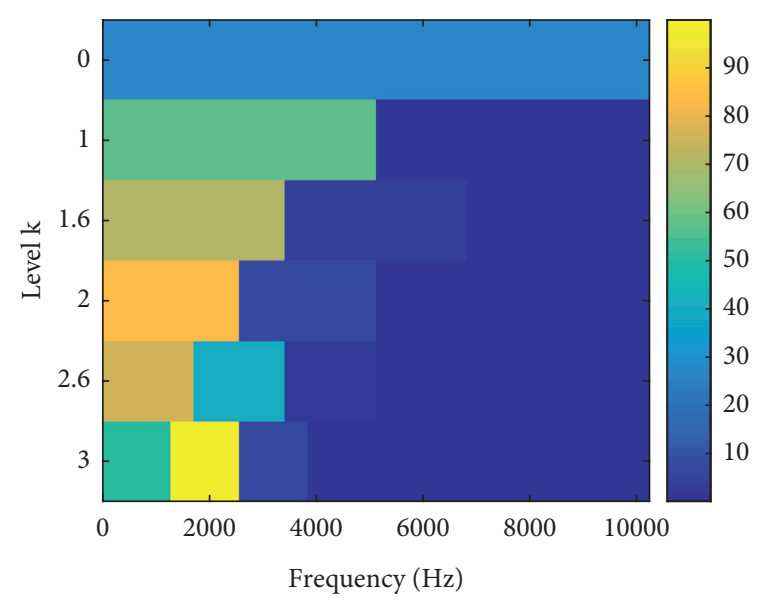

(a)

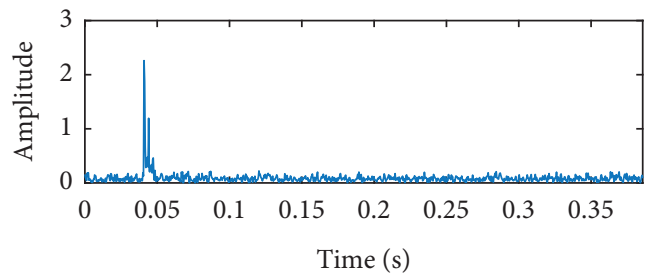

(b)

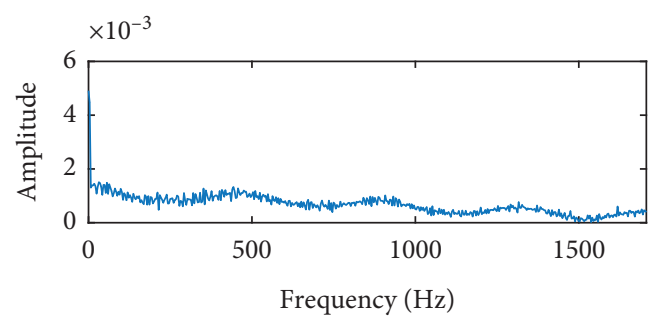

(c)

FIgURE 5: The results of kurtogram on the simulated signal: (a) the kurtogram; (b) the envelope of the filtered signal; (c) the envelope spectrum.

the proposed approach is robust to accidental impulses with high amplitude. The time-domain signal of optimum signal is displayed in Figure 6(b), showing that the impact component has been substantially enhanced. The envelope spectrum can be witnessed in Figure 6(c). It is indicated that the frequency component of $89.6 \mathrm{~Hz}$ is very close to the characteristic frequency $90 \mathrm{~Hz}$ of the inner-race fault, and its harmonics are distinct. Therefore, the simulation results validate the feasibility of the new approach for the extraction of rolling bearing defect from the heavy background noise and large accidental impulses.

One of the main contributions of the proposed approach lies in the fact that CK, which is an optimization index, is used consistently in both pre- and postprocessing stages. This is different from the pieces of the literature available which make use of different optimization indices in different stages [33-37]. To demonstrate the advantages of consistent use of CK in both signal processing stages, CK is substituted 


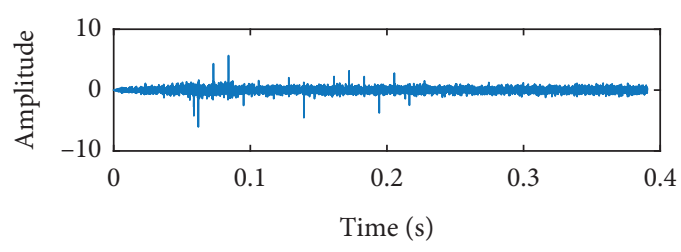

(a)

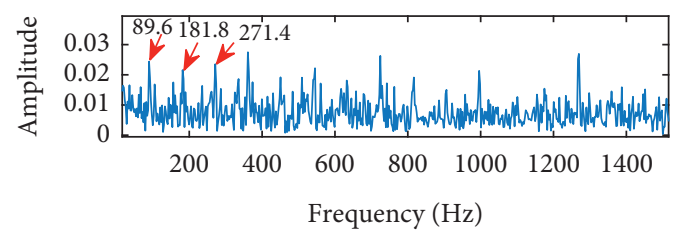

(c)

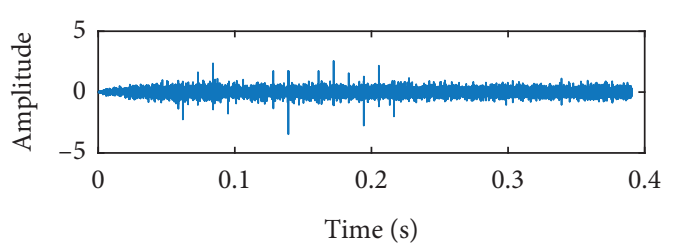

(b)

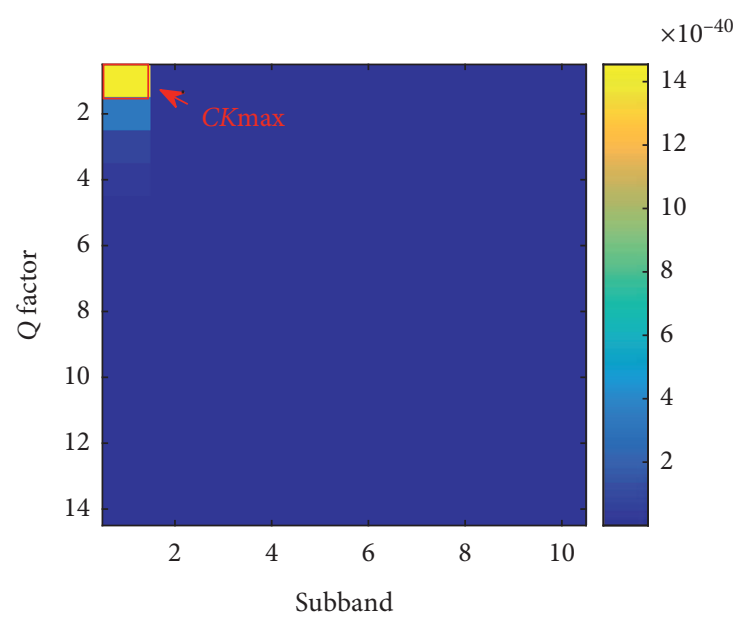

(d)

FIGURE 6: The results of the proposed method on the simulated signal: (a) the preprocessed signal by MCKD; (b) optimal component of the TQWT; (c) the envelope spectrum; (d) the CK map of TQWT.

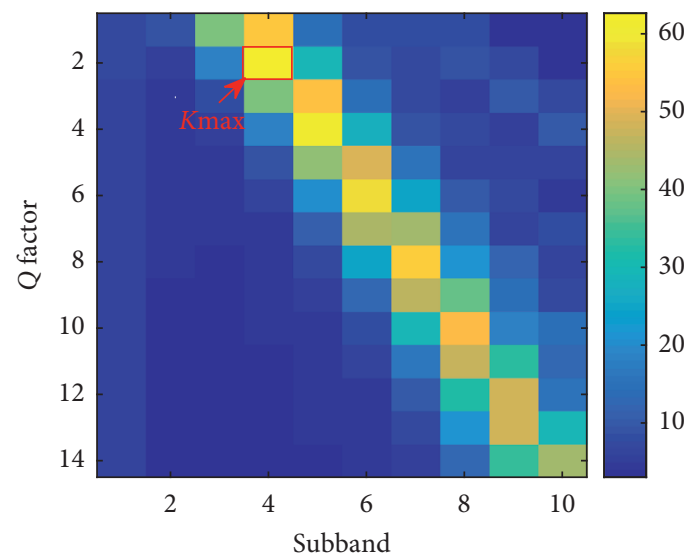

(a)

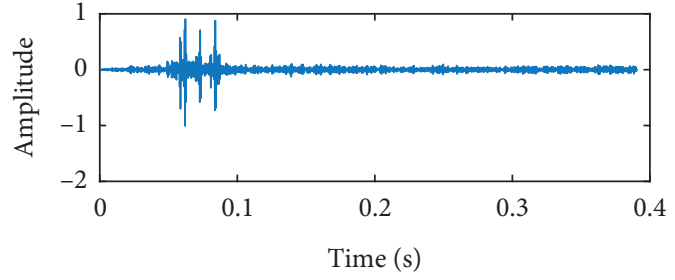

(b)

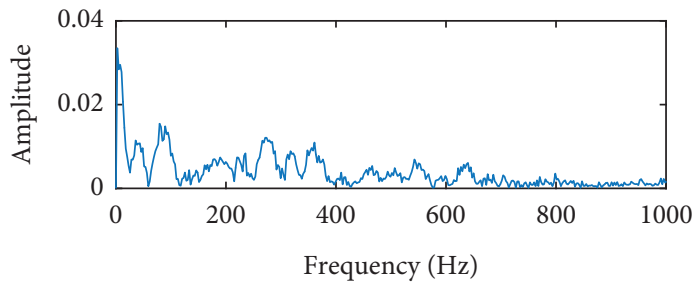

(c)

FIgURE 7: The results of using inconsistent optimization indices on the simulated signal: (a) the kurtosis map of TQWT; (b) the optimal component of TQWT by kurtosis criterion and (c) its envelope spectrum.

by kurtosis in the postprocessing stage to select the best component of TQWT. Figure 7(a) displays the kurtosis values of all TQWT subband components. Based on the criterion of kurtosis maximization, the central frequency $1759 \mathrm{~Hz}$ is selected, which is approximate to the interference impulse frequency of $1500 \mathrm{~Hz}$. The optimal component and 
its envelope spectrum are displayed in Figures 7(b) and 7(c), respectively. There is no dominant fault characteristic frequency component in the envelope spectrum. Therefore, the indispensability of our method is confirmed by the compared method.

\section{Experimental Results and Discussion}

When localized faults occur on bearings, they will induce a series of periodic transient shocks due to vibration responses of mechanical system. Two experimental bearing vibration signals were utilized to illustrate the ability of the proposed method to extract weak periodic transient impulses of an outer-race fault and an inner-race fault in this part. The operating environments of these two cases are different so are the types of rolling bearings. The following Sections 5.1 and 5.2 will illustrate these tests in detail, respectively. To further prove the effectiveness of the presented approach to extract the early fault features, the experimental consequence is contrast with SK and inconsistent optimization indices (kurtosis as the index of TQWT).

\subsection{Experiment I: Bearing Fault with Large Interference.} The first experimental data come from the rotor-bearing fault simulator (test bench built in-house), which can simulate different rolling bearings and rotor faults. The experimental setup is shown in Figure 8, which includes servo motor, motor controller, supporting bearing, bearing house, accelerometer, computer, and data acquisition card. The test bearing type is N205. To simulate the early fault of bearing, one groove on the outer race with $0.1 \mathrm{~mm} \times 0.5 \mathrm{~mm}$ is generated by using a wire cutting machine. The shaft rotating frequency is set at $16.6 \mathrm{~Hz}$. Vibration signals were collected by using the accelerometer at a sampling frequency of $12,000 \mathrm{~Hz}$, and signals of $1 \mathrm{~s}$ length were stored in an industrial computer. According to the computation formulae of fault characteristic frequencies in Table 1, based on the shaft rotating frequency and geometric size of the fault bearing, $f_{o}, f_{i}$, and $f_{b}$ were calculate and presented in Table 2.

The time-domain signal of the bearing outer-race fault is shown in Figure 9(a), where the fault impacts are evident due to regular defect shape and almost without interferences. Therefore, to make the collected vibration signal closer to the real-life operating environment of bearings, the Gaussian random noise of $4 \mathrm{~mm} / \mathrm{s}^{2}$ is added to the collected signal. The compound signal after adding noise is displayed in Figure 9(b). Meanwhile, to simulate the large accidental impulse interference, a random vibration with an amplitude of $60 \mathrm{~mm} / \mathrm{s}^{2}$ is artificially added in the vibration signal between No. 2281-2360 points, and the compound signal is shown in Figure 9(c). The amplitude of accidental impact is much larger than that of bearing fault impacts. The accidental impact is dominant in vibration signals, and the fault characteristics of bearing outer race have been submerged in the noise and interference impact.

The results produced by fast kurtogram for the experimental signal is presented in Figures 10(a)-10(c). The

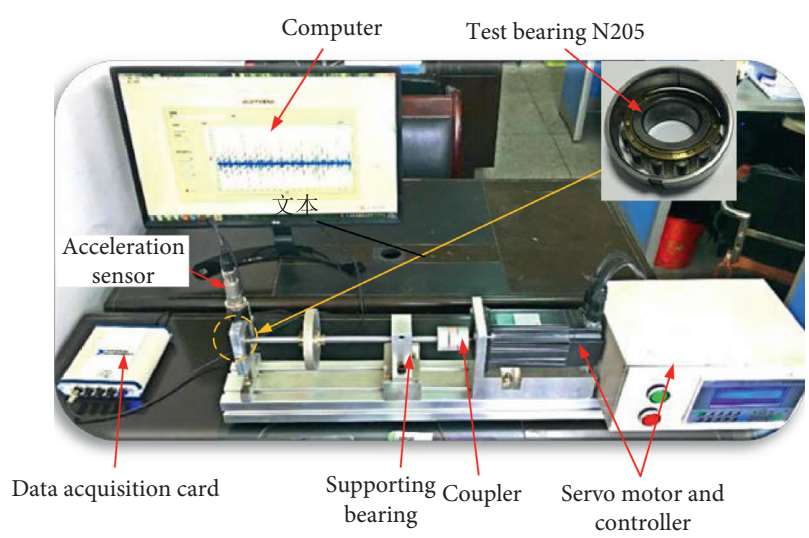

Figure 8: Test bench for bearing fault detection.

diagram of the fast kurtogram is displayed in Figure 10(a), and the filter frequency range is chosen to be [5300, 6000] $\mathrm{Hz}$. However, there is no obvious characteristic frequency component in the envelope spectrum in Figure 10(c). So, the fast Kurtogram fails to detect periodic fault-related characteristics. Therefore, extracting periodic fault features submerged in the strong interference is difficult by using the fast kurtogram.

The proposed approach is used to extract the bearing fault characteristics from the compound signal including Gaussian random noise and large accidental impulses as shown in Figure 9(c). According to the fault characteristic frequencies $f_{o}, f_{i}$, and $f_{b}$, the corresponding period $T_{s}$ of those components are calculated as 93,137 , and 126 points, respectively; therefore, the range of $T$ is set to $[75,150]$. Firstly, MCKD is employed to deconvolute the signal in Figure 9(c). The signal preprocessed by MCKD based on the maximum principle of CK is represented in Figure 11(a), and the optimal period of MCKD is $T=137$, which is consistent with the actual outer period 137 . The periodic transient impulse features of the bearing fault have been initially enhanced, but it is not enough to detect the outerrace defect. Secondly, the pretreated signal induced by MCKD is further decomposed by TQWT, and the first 10 wavelet coefficients are reconstructed by inverse TQWT. The maximum CK in Figure 11(d) implies an optimal frequency range of $[1925,3568] \mathrm{Hz}$ for TQWT. The resultant subsignal and its envelope spectrum are presented in Figures 11(b) and $11(\mathrm{c})$, respectively. The peaks at $88 \mathrm{~Hz}$ and its harmonics $\left(2 f_{o}, 3 f_{o}\right)$ are dominant in Figure 11(c). The result indicates clearly that the outer-race faults disturbed by transmission path, harsh background noise, and large accidental impulses can be extracted effectively by the proposed method.

For comparison, the approach that the optimization index of TQWT in postprocessing stage is replaced by the kurtosis is used to process this fault signal in Figure 9(c) to prove the significance of employing consistently optimization index in the individual stages. Figure 12(a) shows the kurtosis diagram of TQWT. Figures 12(b) and 12(c) express the filtered signal and its envelope spectrum of the selected component according to the kurtosis maximization criterion. There is no any obvious characteristic frequency 
TABLE 1: Computation formulae of fault characteristic frequencies (FCFs).

\begin{tabular}{lc}
\hline Fault characteristic frequency & Formulae \\
\hline Outer-race fault frequency (BPFO) & $f_{o}=(1 / 2) Z(1-(d / D) \cos \alpha) f_{r}$ \\
Inner-race fault frequency (BPFI) & $f_{i}=(1 / 2) Z(1+(d / D) \cos \alpha) f_{r}$ \\
Rolling element fault frequency (BPFR) & $f_{b}=(D / 2 d)\left[1-(d / D)^{2} \cos ^{2} \alpha\right] f_{r}$ \\
Cage defect frequency (CDP) & $f_{c}=(1 / 2)[1-(d / D) \cos \alpha] f_{r}$ \\
\hline
\end{tabular}

$f_{r}, Z, d, D$, and $\alpha$ represent the shaft rotating frequency, the number of balls, the roller diameter, the bearing groove section size, and the bearing pressure angle, respectively.

TABLE 2: Bearing fault characteristic frequencies $(\mathrm{Hz})$ in experiment $\mathrm{I}$.

\begin{tabular}{lccc}
\hline Rotation frequency $f_{r}(\mathrm{~Hz})$ & BPFO $f_{o}(\mathrm{~Hz})$ & BPFI $f_{i}(\mathrm{~Hz})$ & BPFR $f_{b}(\mathrm{~Hz})$ \\
\hline 16.7 & 87.51 & 129.15 & 110.3 \\
\hline
\end{tabular}

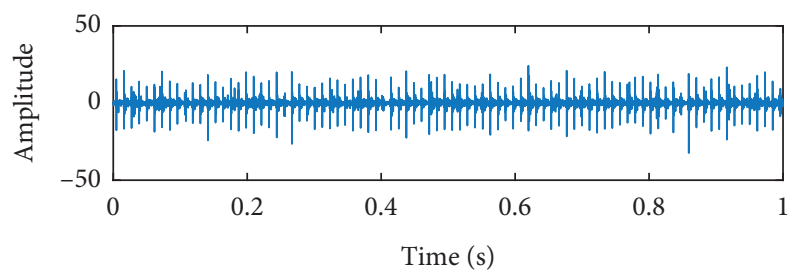

(a)

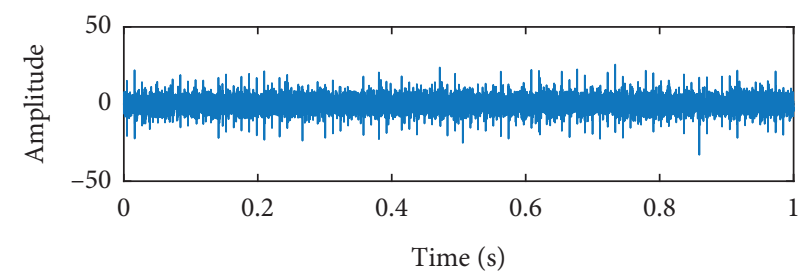

(b)

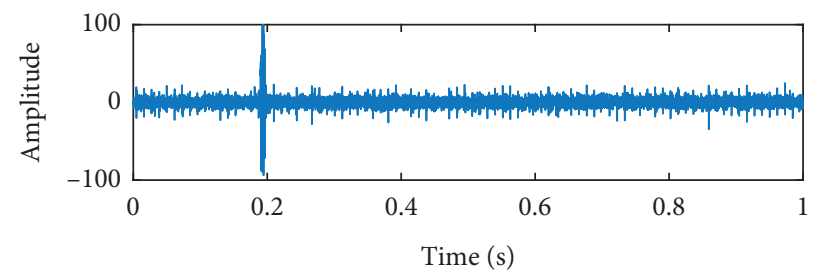

(c)

FIGURE 9: Signals from experiment I: (a) periodic transient impulse signal; (b) added noise signal; (c) added noise and large accidental impulses signal.

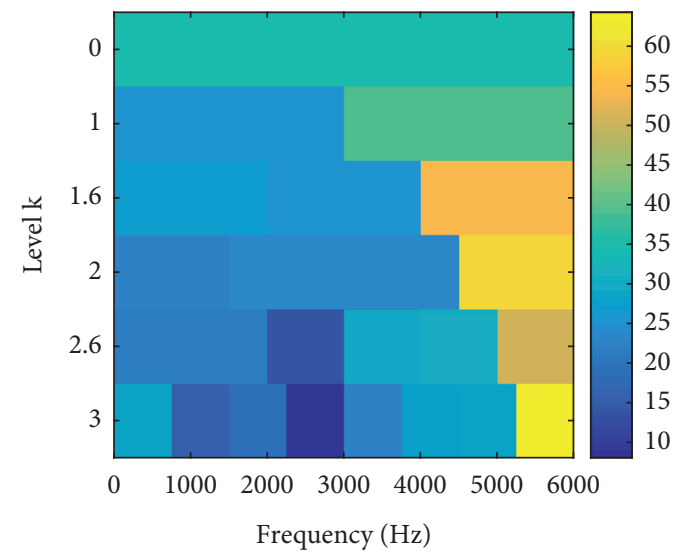

(a)

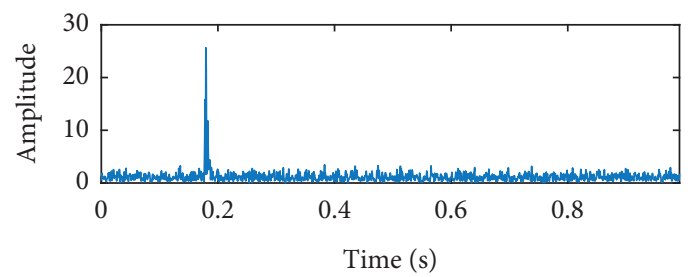

(b)

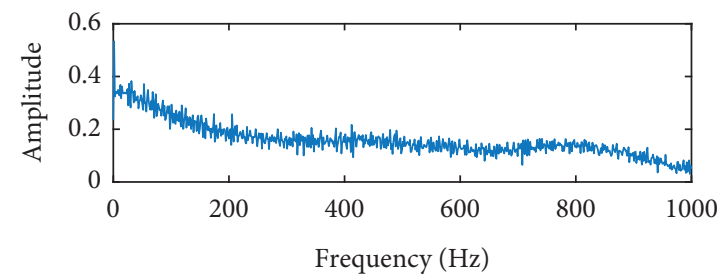

(c)

FIGURE 10: The results of experiment I by using SK: (a) the kurtogram; (b) the envelope of the filtered signal and (c) its envelope spectrum. 


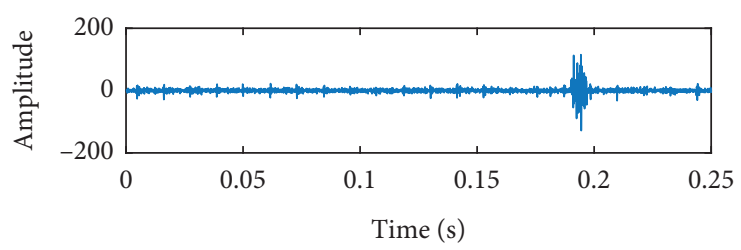

(a)

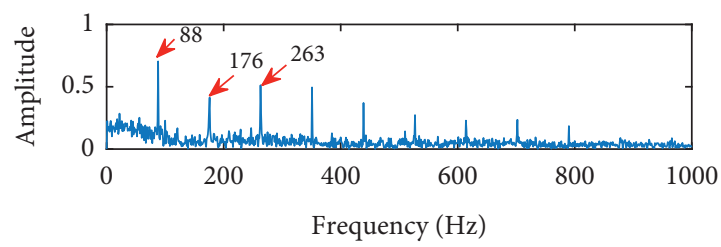

(c)

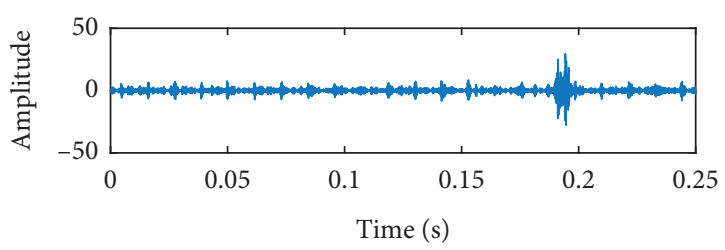

(b)

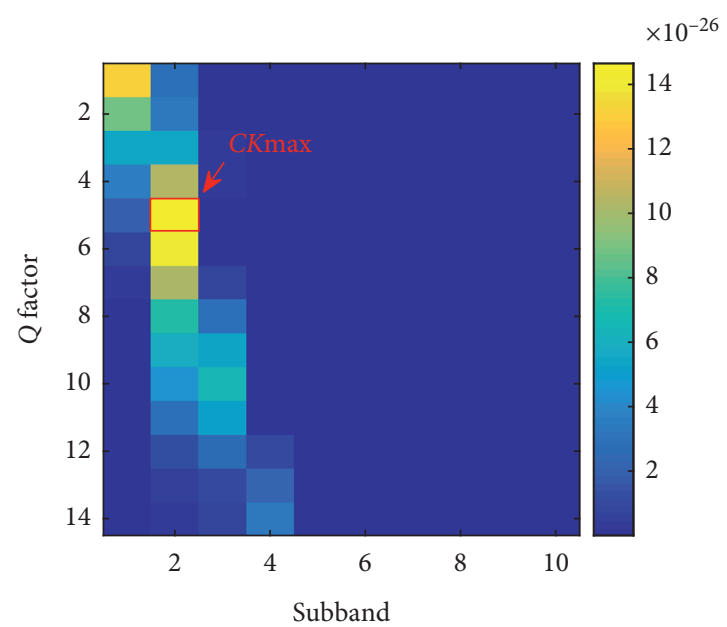

(d)

FIGURE 11: The results by employing the proposed approach: (a) the preprocessed signal by MCKD; (b) optimal component of the TQWT; (c) its envelope spectrum; (d) the CK map of TQWT.

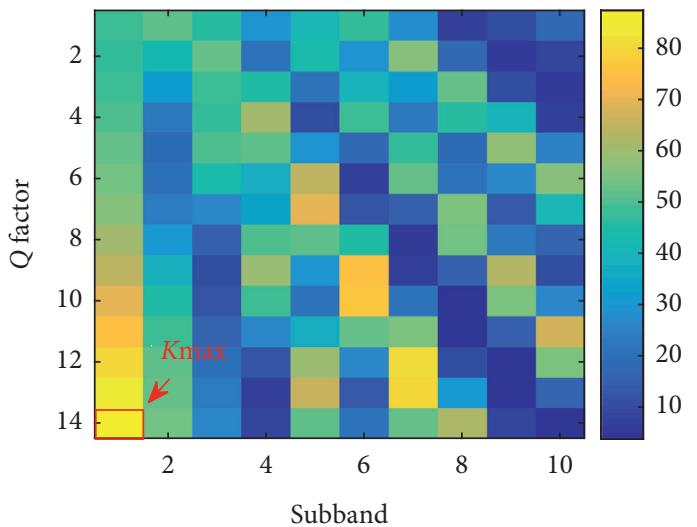

(a)

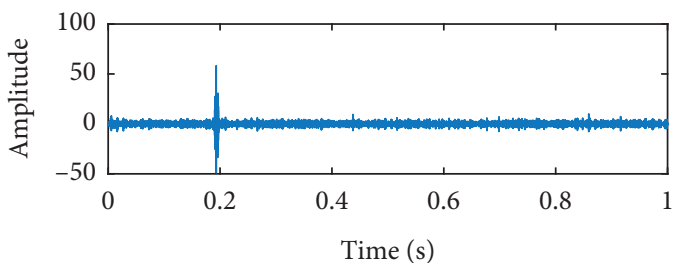

(b)

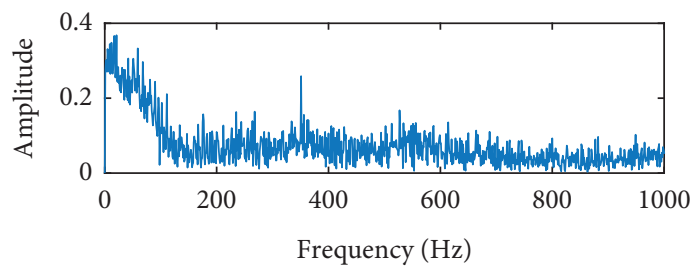

(c)

FIGURE 12: The analyzed result by employing the comparison approach: (a) the kurtosis map of TQWT; (b) the filtered signal and (c) its envelope spectrum. 


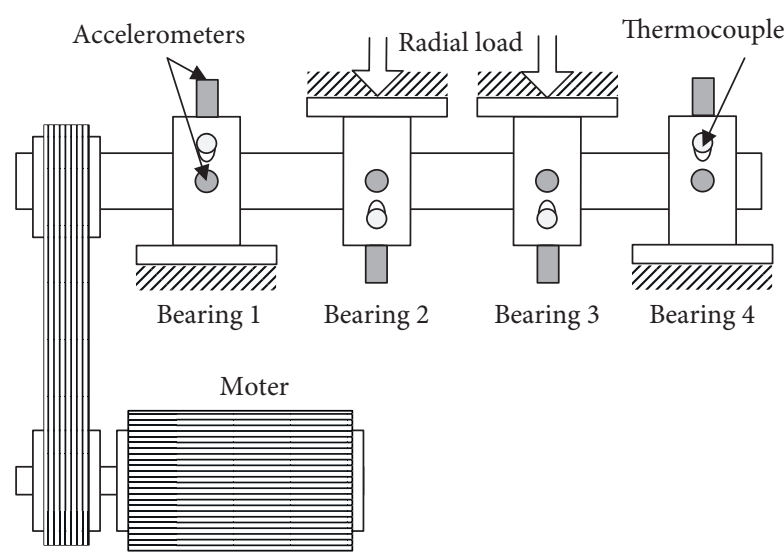

(a)

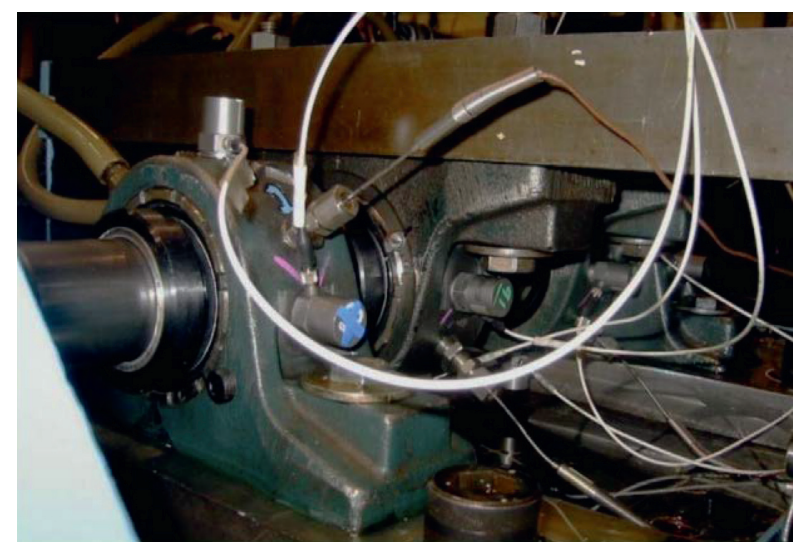

(b)

FIgUre 13: Run-to-failure test bench of rolling bearings. (a) Schematic of the run-to-failure test rig; (b) local photo.

TABLE 3: Fault characteristic frequencies $(\mathrm{Hz})$ in experiment II.

\begin{tabular}{lccc}
\hline Rotation frequency $f_{r}(\mathrm{~Hz})$ & BPFO $f_{o}(\mathrm{~Hz})$ & BPFI $f_{i}(\mathrm{~Hz})$ & BPFR $f_{b}(\mathrm{~Hz})$ \\
\hline 33.3 & 236.4 & 296.9 & 139.9 \\
\hline
\end{tabular}

component, so that it is impossible to extract the fault impulses. In addition, this method confirms the necessity of consistently using CK as an optimization index in the individual stages for the extraction of a rolling bearing fault feature.

5.2. Experiment II: Early Fault Detection in Bearing. The second experiment data were acquired from NSSA. It was performed by IMS of the University of Cincinnati, USA, and the run-to-failure test bench is displayed in Figure 13 [23]. The test system consists of the motor, spindle, bearing housings, and belt drive. Four double row roller bearings of type Rexnord ZA-2115 are installed on the spindle. To shorten the fatigue time span, the radial force of 6000 pounds was loaded on the top surface of bearing housing 2 and 3 , and the whole life experiment lasted 7 days. Then, the test bench was disassembled, and the serious fault on the outer race of bearing 1 was found. Based on the theoretical calculation, the fault characteristic frequency is $236.4 \mathrm{~Hz}$ as shown in Table 3.

During this experiment, the signal was obtained every 10 minutes, and the sampling frequency is $20 \mathrm{kHz}$. A total of 984 data files were collected during the experiment, and each data file contains four columns pertinent to the four acceleration sensors on the four bearing housings. The data in the first column that were explored as bearing 1 failed finally. Root mean square (RMS) is a widespread index to indicate the degradation processing of bearings. The RMS of the data in column 1 of all the 984 data files is shown in Figure 14(a), and its value rises weakly at $534^{\text {th }}$ file. Therefore, it can be considered that the early defect happens at No. 534, of which the signal and its envelope spectrum are plotted in Figure $14(\mathrm{~b})$ and $14(\mathrm{c})$, respectively. There is a $230.5 \mathrm{~Hz}$ frequency component in Figure 14(c) but without harmonic components, which is unable to guarantee a sure diagnosis of outer-race fault.

The kurtogram of the No. 534 signal is shown in Figure 15(a). The central frequency of the selected filter bandwidth is $7500 \mathrm{~Hz}$, and its bandwidth is $1666 \mathrm{~Hz}$. The envelope spectrum of the filtered signal shown in Figure 15(c) reveals only one frequency component of $230.4 \mathrm{~Hz}$ and there are no explicit harmonics frequency components. Like the envelop spectrum in Figure 14(c), SK cannot make a sure diagnosis of early bearing faults.

In the case of our method, the range of period $T$ is first set to $[65,110]$ based on the fault characteristic frequencies in Table 3. MCKD is then adopted to preprocess the original signal with the result shown in Figure 16(a), which demonstrates that the influence of transmission path has been alleviated to a certain extent. The first 10 subband components of TQWT under various $Q$ factors are reconstructed, and their $\mathrm{CK}$ values are imaged in Figure $16(\mathrm{~d})$. The reconstructed subsignal with maximum $\mathrm{CK}$, and its envelope spectrum is displayed in Figures 16(b) and 16(c), respectively. In Figure 16(c), the frequency component of $232 \mathrm{~Hz}$ and its harmonics $(460 \mathrm{~Hz}, 690 \mathrm{~Hz})$ are distinct. Although $232 \mathrm{~Hz}$ is slightly different from the theoretical calculation results $(236.5 \mathrm{~Hz})$, it is enough to signal that the localized fault occurs on the outer race at the moment. The deviation between the actual frequency and the theoretical fault characteristic frequency may be attributed to the fluctuation of rotational speed and the slippage of internal components of bearings. This case illustrates that the proposed approach has certainly feasibility and superiority to detect bearing early weak fault under severe obstacles of the transmission path and strong background noise. 


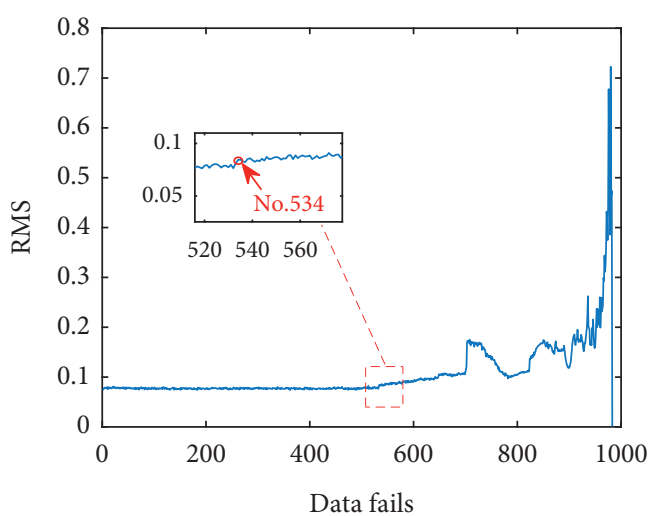

(a)

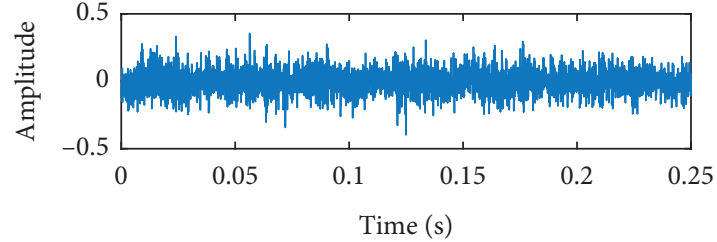

(b)

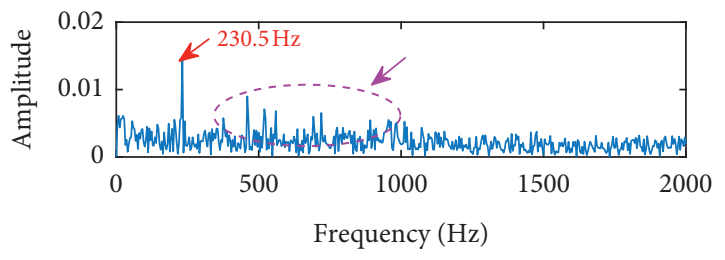

(c)

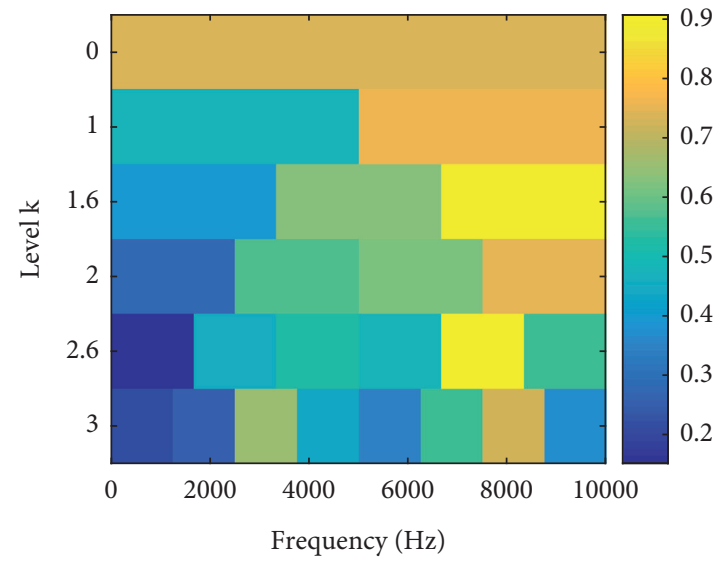

(a)

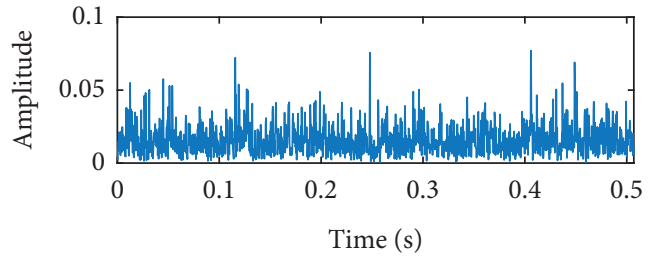

(b)

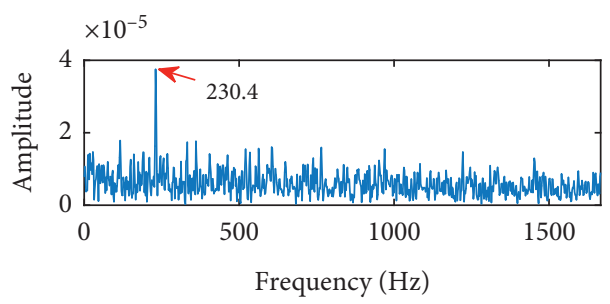

(c)

FIGURE 15: The results on experiment II by employing SK: (a) the kurtogram; (b) the envelope of the filtered signal and (c) its envelope spectrum. 


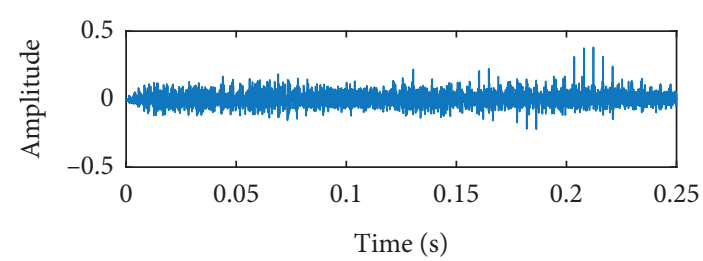

(a)

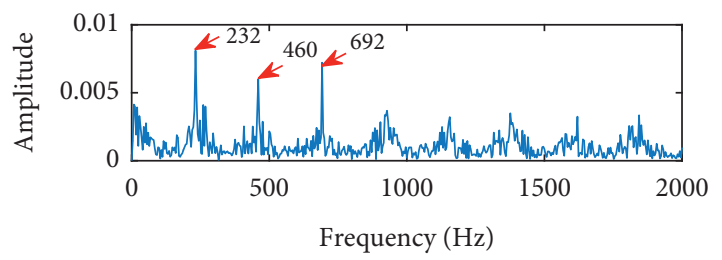

(c)

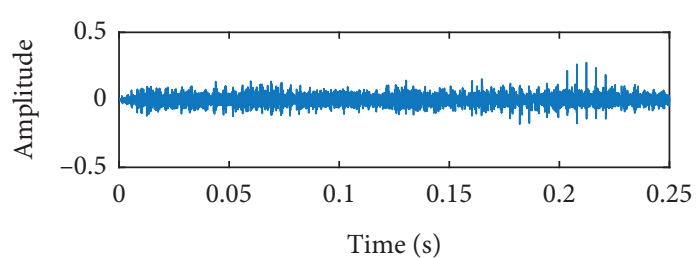

(b)

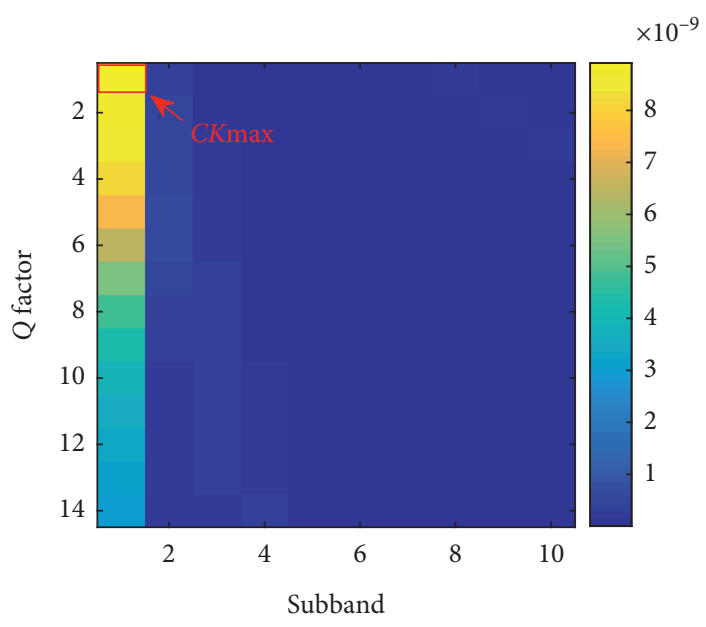

(d)

FIGURE 16: The results using the proposed approach: (a) the preprocessed signal by MCKD; (b) optimal component of the TQWT; (c) its envelope spectrum and (d) the CK map of TQWT.

\section{Conclusions}

The extraction of periodic transient impulses plays a crucial role in bearing fault diagnosis. At present, the majority of the optimization index used in signal processing approaches fails to consider the periodical occurrence of the fault impacts of rolling bearing. Moreover, existing hybrid diagnosis paradigm exploits different optimization index in individual signal processing stage. Aimed at such problems, a multistage fault feature extraction method is proposed in the present research, which takes correlation kurtosis as the optimization index in both MCKD preprocessing and TQWT postprocessing. It is expected to reduce the influence of transmission path, background noise, and large accidental impulses and thus ensure an enhancement of localized bearing fault induced periodical impacts. The proposed method was validated on the simulation signal, laboratory signal, and fatigue test data and achieved a better performance than other methods like kurtogram in the extraction of weak fault features. The primary works and contributions of this paper are as follows:

(1) Consistently using $\mathrm{CK}$ as the optimization index in both two stages of the proposed hybrid diagnosis approach assure a consistent optimization toward relieving the effect of signal transmission path, background noise, and accidental external shocks.

(2) The problem with fault period $T$ in MCKD which depends heavily on prior knowledge is addressed by CK maximization on the deconvoluted signals with a prescribed range of $T$ with consideration of factors like speed fluctuations.

(3) The comparison with commonly used kurtogram and a hybrid diagnosis with inconsistent optimization indices in individual stages demonstrate the advantages of the proposed method.

\section{Data Availability}

The run-to-failure experiment data of rolling bearing can be obtained from shttp://www.imscenter.net/IMS/news.

\section{Conflicts of Interest}

The authors declare that there are no conflicts of interest regarding the publication of this paper.

\section{Acknowledgments}

This work was supported by the National Science Foundation of China (Nos. 51665013 and 51865010), the Natural Science Foundation of Jiangxi Province (Nos. 20171BAB206028 and 20171BAB216030), and the Jiangxi Province Graduate Student Innovation Project (Nos. YC2018-S248 and YC2019-S243).

\section{References}

[1] Z. Ma, C. Gu, and Y. Li, “Application of improved resonance demodulator in rolling bearing fault diagnosis," Journal of 
Electronic Measurement \& Instrumentation, vol. 97, no. 7, pp. 213-235, 2016.

[2] Y. Xu, Z. Fan, and K. Zhang, "A novel method for extracting maximum kurtosis component and its applications in rolling bearing fault diagnosis," Shock and Vibration, vol. 2019, no. 35, Article ID 8218237, 2019.

[3] A. Glowacz, W. Glowacz, and Z. Glowacz, "Early fault diagnosis of bearing and stator faults of the single-phase induction motor using acoustic signals," Measurement, vol. 113, no. 3, pp. 1-9, 2018.

[4] Y. Kozik, M. Xu, and R. Wang, "A fault diagnosis scheme for rolling bearing based on local mean decomposition and improved multiscale fuzzy entropy," Journal of Sound and Vibration, vol. 360, no. 60, pp. 277-299, 2016.

[5] L. Huang, H. Su, and Y. Li, "Research on extraction of compound fault characteristics for rolling bearings in wind turbines," Entropy, vol. 22, no. 6, pp. 682-715, 2020.

[6] A. Rai and S. H. Upadhyay, "A review on signal processing techniques utilized in the fault diagnosis of rolling element bearings," Tribology International, vol. 96, no. 6, pp. 289-306, 2016.

[7] Y. Cheng, B. Chen, G. Mei, Z. Wang, and W. Zhang, “A novel blind deconvolution method and its application to fault identification," Journal of Sound and Vibration, vol. 460, Article ID 114900, 2019.

[8] S. Wan and X. Zhang, "Teager energy entropy ratio of wavelet packet transform and its application in bearing fault diagnosis," Entropy, vol. 20, no. 5, pp. 11-49, 2018.

[9] L. Jiang, S. Guo, and V. V. Silberschmidt, "Modified kernel marginal fisher analysis for feature extraction and its application to bearing fault diagnosis," Shock and Vibration, vol. 2016, Article ID 1205868, 16 pages, 2016.

[10] D. Zhen, J. Guo, Y. Xu et al., "A novel fault detection method for rolling bearings based on non-stationary vibration signature analysis," Sensors, vol. 19, no. 18, pp. 39-94, 2019.

[11] Z. Liu, J. Ding, J. Lin, and Y. Huang, "A rolling bearing fault diagnosis-optimized scale-space representation for the empirical wavelet transform," Shock and Vibration, vol. 2018, Article ID 2749689, 2018.

[12] Y. Miao, M. Zhao, and J. Lin, "Periodicity-impulsiveness spectrum based on singular value negentropy and its application for identification of optimal frequency band," IEEE Transactions on Industrial Electronics, vol. 66, no. 4, pp. 3127-3138, 2019.

[13] C. Delpha, D. Diallo, and N. Moubayed, "Multiple incipient fault diagnosis in three-phase electrical systems using multivariate statistical signal processing," Engineering Applications of Artificial Intelligence, vol. 73, no. 3, pp. 68-79, 2018.

[14] T. Wang, F. Chu, and Y. Kong, "Compound faults detection in gearbox via meshing resonance and spectral kurtosis methods," Journal of Sound and Vibration, vol. 392, no. 2, pp. 367-381, 2017.

[15] Z. Ma, F. Lu, and X. Li, "An adaptive generalized demodulation method for multimedia spectrum analysis is applied in rolling bearing fault diagnosis," IEEE Access, vol. 8, no. 19, pp. 20687-20699, 2020.

[16] G. Cai, X. Chen, and Z. He, "Sparsity-enabled signal decomposition using tunable $Q$-factor wavelet transform for fault feature extraction of gearbox," Mechanical Systems and Signal Processing, vol. 41, no. 1-2, pp. 34-53, 2013.

[17] R. A. Wiggins, "Minimum entropy deconvolution," Geophysical Prospecting for Petrole, vol. 16, no. 1, pp. 21-35, 1980.

[18] Y. Gao, M. Karimi, and A. A. Kudreyko, "Spare optimistic based on improved ADMM and the minimum entropy de- convolution for the early weak fault diagnosis of bearings in marine systems," ISA Transactions, vol. 22, no. 13, pp. 10474-14025, 2017.

[19] J. Li, M. Li, and J. Zhang, "Rolling bearing fault diagnosis based on time-delayed feedback monostable stochastic resonance and adaptive minimum entropy deconvolution," Journal of Sound and Vibration, vol. 401, no. 1, pp. 139-151, 2017.

[20] X. Jiang, X. Cheng, W. Huang, C. Shen, and Z. Zhu, "A new 10norm embedded MED method for roller element bearing fault diagnosis at early stage of damage," Measurement, vol. 127, no. 7, pp. 414-424, 2018.

[21] F. Wang, C. Liu, and W. Su, "Combined failure diagnosis of slewing bearings based on MCKD-CEEMD-ApEn," Shock and Vibration, vol. 2018, Article ID 6321785, 2018.

[22] Z. J. Wang, J. Zhou, J. Y. Wang et al., “A novel fault diagnosis method of gearbox based on maximum kurtosis spectral entropy deconvolution," IEEE Access, vol. 7, pp. 29520-29532, 2019.

[23] Y. Miao, M. Zhao, J. Lin, and Y. Lei, "Application of an improved maximum correlated kurtosis deconvolution method for fault diagnosis of rolling element bearings," Mechanical Systems and Signal Processing, vol. 92, no. 2, pp. 173-195, 2017.

[24] Y. Cheng, Z. Wang, and W. Zhang, "Particle swarm optimization algorithm to solve the deconvolution problem for rolling element bearing fault diagnosis," ISA Transaction, vol. 2, no. 17, pp. 634-649, 2019.

[25] G. L. McDonald, Q. Zhao, and M. J. Zuo, "Maximum correlated kurtosis deconvolution and application on gear tooth chip fault detection," Mechanical Systems and Signal Processing, vol. 33, no. 3, pp. 237-255, 2012.

[26] X. Lyu, Z. Hu, H. Zhou, and Q. Wang, "Application of improved MCKD method based on QGA in planetary gear compound fault diagnosis," Measurement, vol. 139, no. 9, pp. 236-248, 2019.

[27] Z. Huo, Y. Zhang, P. Francq, L. Shu, and J. Huang, "Incipient fault diagnosis of roller bearing using optimized wavelet transform based multi-speed vibration signatures," IEEE Access, vol. 9, no. 25, pp. 99-112, 2017.

[28] X. Chen, F. Feng, and B. Zhang, "Weak Fault feature extraction of rolling bearings based on an improved kurtogram," Sensors, vol. 16, no. 9, pp. 1482-1501, 2016.

[29] J. Duan, T. Shi, J. Xuan, and Y. Zhang, "Multiband envelope spectra extraction for fault diagnosis of rolling element bearings," Sensors, vol. 18, no. 5, pp. 1466-1478, 2018.

[30] J. Antoni, "Fast computation of the kurtogram for the detection of transient faults," Mechanical Systems and Signal Processing, vol. 21, no. 1, pp. 108-124, 2007.

[31] X. Zhang, J. S. Kang, L. Xiao, J. Zhao, and H. Teng, “A new improved kurtogram and its application to bearing fault diagnosis," Shock and Vibration, vol. 2015, Article ID 385412, 2015.

[32] I. W. Selesnick, "Wavelet transform with tunable Q-factor," IEEE Transactions on Signal Processing, vol. 59, no. 8, pp. 3560-3575, 2011.

[33] Z. Shang, G. Rui, L. Cheng et al., "Rolling bearing fault diagnosis method based on MOMEDA and IEWT," International Journal of Information and Management Sciences, vol. 29, no. 4, pp. 345-363, 2018.

[34] Y. Zhang, Z. Fan, and X. Gao, "A fault diagnosis method of train wheelset rolling bearing combined with improved LMD and FK," Sensors, vol. 62, no. 7, pp. 335-351, 2019.

[35] Y. Li, X. Liang, M. Xu, and W. Huang, "Early fault feature extraction of rolling bearing based on ICD and tunable Q- 
factor wavelet transform," Mechanical Systems and Signal Processing, vol. 86, no. 6, pp. 204-223, 2017.

[36] P. Ma, H. Zhang, W. Fan, and C. Wang, "Early fault diagnosis of bearing based on frequency band extraction and improved tunable Q-factor wavelet transform," Measurement, vol. 137, no. 7, pp. 189-202, 2019.

[37] W. Cai, Z. J. Yang, Z. J. Wang et al., "A new compound fault feature extraction method based on multipoint kurtosis and variational mode decomposition," Entropy, vol. 20, no. 7, pp. 5-21, 2018.

[38] J. Zhang, J. Zhang, and M. Zhong, "Detection for incipient damages of wind turbine rolling bearing based on VMDAMCKD method," IEEE Access, vol. 8, no. 12, pp. 27-46, 2019.

[39] I. W. Selesnick, "Resonance-based signal decomposition: a new sparsity-enabled signal analysis method," Signal Processing, vol. 91, no. 12, pp. 2793-2809, 2011.

[40] I. Bayram and I. W. Selesnick, "Frequency-domain design of overcomplete rational-dilation wavelet transforms," IEEE Transactions on Signal Processing, vol. 57, no. 8, pp. 29572972, 2009.

[41] I. Bayram and I. W. Selesnick, "Overcomplete discrete wavelet transforms with rational dilation factors," IEEE Transaction Signal Process, vol. 57, no. 1, pp. 131-145, 2008. 